\title{
Editorial
}

Gefässchirurgie 2016 $21: 72-73$

DOI 10.1007/s00772-016-0120-3

๑) Springer-Verlag Berlin Heidelberg 2016

CrossMark

Die akute Ischämie stellt den Gefäßmediziner immer wieder vor große Herausforderungen. Dabei scheint die Diagnostik mit den Kardinalzeichen in Stein gemeißelt. In meiner ChirurgieVorlesung hießen die Symptome - selbstverständlich - die 6 P nach Pratt. Niemals habe ich mich gefragt, - war es denn Pratt, der diese Zeichen als erster benannt hat? Oder wer war denn Pratt überhaupt? Und ist das der gleiche Pratt mit den Warnvenen bei der Thrombose?

Wenn man bedenkt, wie häufig der Name im klinischen Alltag bei uns fällt, ist es erstaunlich, wie wenig Informationen man über diesen Chirurgen findet. Gibt man seinen kompletten Namen: „Gerald Hillary Pratt" in Google ein, wird man mitnichten einen Lebenslauf finden. Allenfalls Hinweise auf sein Buch „Surgical Management of Vascular Diseases" aus dem Jahr 1949 werden aufgelistet. Pratt wurde 1903 geboren. Laut Internet war er der Mannschaftsarzt des New York Giants Football und des Yankees Baseball Teams. Er praktizierte am St. Vincents-Hospital in New York (geschlossen 2010) und war wohl seinerzeit ein bekannter kardiovaskulärer Chirurg. Angeblich hat er u. a. die Königin von England behandelt [3].

Wie dem auch sei, es ist immer interessant, sich bis zur Quelle durchzuarbeiten. Ohne zu sehr Kritik an den eigenen Autoren zu äußern - die Zitation „Pratt, G.H.: Cardio vascular Surgery. London Kimpton (1954)" ist nicht sehr hilfreich.

Dabei ist es viel einfacher. G.H. Pratt hat 1954 einen Artikel im American Journal of Surgery publiziert [2]. Dort heißt es: „It can be diagnosed usually on the history alone. The signs (six „P“ signs) paleness, pulselessness, parasthesia, paralysis, pain and prostration are apparent. (Table ii.)“. Somit kann also bestätigt werden, dass Pratt diese Zeichen als erster zusammengefasst und 1954 publiziert hat.

\author{
A. Larena-Avellaneda \\ Klinik und Poliklinik für Gefäßmedizin, Universitäres Herzzentrum Hamburg, \\ Universitätsklinikum Hamburg-Eppendorf, Hamburg, Deutschland
}

\section{Die 6-P-Zeichen nach Pratt}

In seiner Arbeit über die Behandlung verschlossener Arterien (JAMA 1946) beschreibt er die Klinik noch anders: „The sudden pain, inability to use the part, coldness and a whiteness of the limb in the presence of auricular fibrillation are diagnostic“.

\section{》) Pratt hat die 6-P-Zeichen als erster zusammengefasst und 1954 publiziert}

Sucht man heute im Internet nach den $6 \mathrm{P}$ der akuten Ischämie, wird international der Name Pratt nicht erwähnt. Das sechste P („Prostration“), welches heutzutage ja zum Glück nicht mehr so oft gefunden wird, wird in Wikipedia mit "Poikilothermie" angegeben - und dies zitiert aus einem Fachartikel [1]. Dies finde ich bemerkenswert, wird doch mit diesem Begriff die Wechselwarmblütigkeit definiert. Da gefiel mir „perishingly cold“" schon besser, auf diese Bezeichnung trifft man auch recht häufig und sie beschreibt das Krankheitsbild sehr treffend.

Die heutige Ausgabe hat sich der akuten kritischen Ischämie gewidmet - ein sehr wichtiges Thema mit einer großen Option an Behandlungsmöglichkeiten. Die klinische Bedeutung gerade in der Lehre kann nicht genug betont werden. In vielen Kliniken gibt es große, interdisziplinäre Notaufnahmen, und es ist wichtig, genau diese Patienten, bei denen jede Minute die Prognose der Extremität verschlechtert, sehr rasch der richtigen Fachdisziplin zuzuführen. Auf der anderen Seite sollte es jedem approbierten Arzt auch möglich sein, auf eine Konsilanforderung mit der Frage auf akuten arteriellen Verschluss nachts um 3 zu verzichten, wenn der Patient einen tastbaren Fußpuls hat.
In diesem Sinne wünsche ich Ihnen viel Freude bei der Lektüre dieser Ausgabe.

Herzlichst,

Ihr

Axel Wena - Mvellande

Prof. Dr. A. Larena-Avellaneda

\section{Korrespondenzadresse}

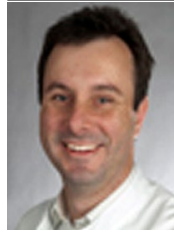

Prof. Dr. A. Larena-

Avellaneda

Klinik und Poliklinik für

Gefäßmedizin,

Universitäres Herzzentrum

Hamburg, Universitätsklinikum Hamburg-Eppendorf Martinistr. 52, 20246 Hamburg larena@uke.de

\section{Literatur}

1. Brearley S (2013) Acute leg ischaemia. BMJ 346:f2681

2. Pratt GH, Krahl E (1954) Surgical therapy for the occluded artery. Am J Surg 87 (5):722-729

3. http://www.zoominfo.com/p/GeraldPratt/1445607830 
Hier steht eine Anzeige.

\section{算 Springer}

